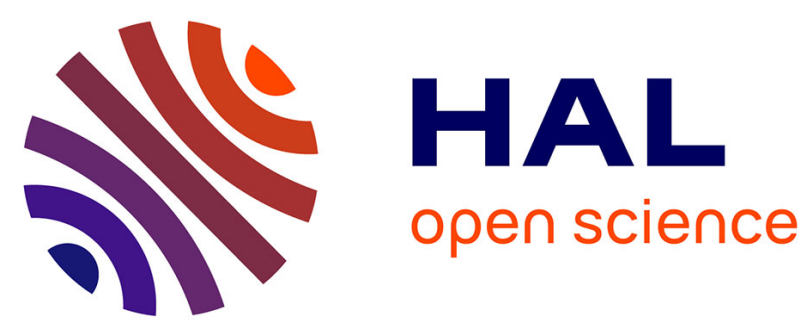

\title{
Impact of the closed-loop phase shift on the frequency stability of capacitive MEMS oscillators
}

Alexis Brenes, Jérôme Juillard, Filipe Vinci dos Santos

\section{To cite this version:}

Alexis Brenes, Jérôme Juillard, Filipe Vinci dos Santos. Impact of the closed-loop phase shift on the frequency stability of capacitive MEMS oscillators. 2016 Symposium on Design, Test, Integration and Packaging of MEMS/MOEMS (DTIP), May 2016, Budapest, Hungary. pp.169-172, 10.1109/DTIP.2016.7514862 . hal-01357847

\section{HAL Id: hal-01357847}

https://hal-centralesupelec.archives-ouvertes.fr/hal-01357847

Submitted on 30 Aug 2016

HAL is a multi-disciplinary open access archive for the deposit and dissemination of scientific research documents, whether they are published or not. The documents may come from teaching and research institutions in France or abroad, or from public or private research centers.
L'archive ouverte pluridisciplinaire HAL, est destinée au dépôt et à la diffusion de documents scientifiques de niveau recherche, publiés ou non, émanant des établissements d'enseignement et de recherche français ou étrangers, des laboratoires publics ou privés. 


\title{
Impact of the closed-loop phase shift on the frequency stability of capacitive MEMS oscillators
}

\author{
A. Brenes ${ }^{1,2,3}$ J. Juillard ${ }^{2}$ and F. Vinci dos Santos $^{3}$ \\ ${ }^{1}$ THALES Avionics \\ Valence, France \\ alexis.brenes@centralesupelec.fr \\ ${ }^{2}$ GeePs | Group of electrical engineering - Paris, UMR CNRS 8507, CentraleSupélec, Univ. Paris-Sud, Sorbonne Universités, \\ UPMC Univ. Paris 06, 3,11 rue Joliot-Curie, Plateau de Moulon F-91192 Gif-sur-Yvette CEDEX. \\ ${ }^{3}$ CentraleSupélec Advanced Analog Design Chair, 3 rue Joliot-Curie, 91192 Gif-sur-Yvette, France
}

\begin{abstract}
Phase noise is a clue performance indicator for MEMS-based resonant sensors. The optimal resolution achievable with these sensors is limited by the close-to-the-carrier phase noise resulting from the modulation of noise sources by the mechanical resonator. In this paper, we focus on the effect of a white noise input source on a one-sided capacitive MEMS resonator. We study how the closedloop phase shift affects its frequency stability. Our study reveals the existence of optimal points, with phase noise minimization and SNR maximization, which cannot be predicted by a traditional third-order Taylor-Series approach. We reveal that, due to the modulation of the actuation force by the electrostatic nonlinearity, tuning the closed-loop phase shift to improve the oscillator frequency stability is especially relevant.
\end{abstract}

Keywords - Electrostatic nonlinearity, closed-loop systems, oscillators, frequency stability, capacitive MEMS.

\section{INTRODUCTION}

MEMS resonant sensors are known for their high resolution achievable with small and relatively cheap systems [1] [2]. In these sensors, the measurement process is based on the dependency of the natural frequency of the resonator on the measured quantity. One solution to track this natural frequency is to place the resonator in a self-oscillating loop. However, the resolution of the sensors based on this principle depends on the frequency stability of the closed loop in the presence of noise sources.

Several works have already addressed theories of phase noise in oscillators [3] [4] [5]. The phase noise of MEMS oscillators results from noise injection at different stages, such as the input of the resonator or the feedback electronics [3]. The spectrum of an oscillator is expected to be made of two principal components. One component results from additive white noise at frequencies close to the carrier and eventually the effect of nonlinearities which mix the noise at other frequencies into the pass band of interest. The other main component is due to the long-term parameter variation linked to several reliability issues such as dielectric charging, creep or fatigue [6]. These latter effects are outside the scope of this paper. In this study, we will only focus on the effect of additive white noise introduced by random processes such as thermal fluctuations.

The maximal resolution achievable with a sensor is fundamentally limited by the conversion of amplitude noise into phase noise through the electromechanical transduction [7]. To estimate this optimal performance, a precise model of the resonator is required. Among the studies of phase noise, some have already addressed phase noise models for closed-loop electronic MEMS oscillators [8] [9] [10]. One recent study has even focused on the impact of the phase introduced in the feedback loop on the frequency stability of oscillators subject to cubic hardening restoring forces [11]. However, the effects of the electrostatic nonlinearity on capacitive MEMS resonators induce effects which cannot be explained by the presence of a cubic nonlinear force, neither in open-loop [12], nor in closed-loop systems [13]. None of the aforementioned articles have studied the impact of the feedback phase shift on the frequency stability of oscillators based on capacitive MEMS resonators. This is the main purpose of this paper.

In this study, we estimate the performance of a nonlinear MEMS-based oscillator in terms of frequency stability by computing the variance of the frequency variations like in the work of Sancho et al. [14]. Compared to them, we derive a simple closed-form expression of this variance valid for high-Q MEMS-based oscillators. Finally, we adapt these expressions to capacitive MEMS resonators, which are subject to electrostatic softening.

This paper is organized as follows: in Section II, we adapt the theory given by Sancho et al. [14] to a resonator actuated by a linear force but subject to a nonlinear restoring force. We illustrate this approach on a MEMS resonator affected by cubic hardening and study the impact of the operating point on the frequency stability. Finally, in Section III, we generalize this method to a resonator subject to a nonlinear actuation force and take the example of a capacitive MEMS resonator.

\section{FREQUENCY STABILITY ESTIMATION}

In this section, we study the case of a MEMS-based sine-wave oscillator whose resonator is subject to a nonlinear restoring force. The actuation force is firstly assumed to be displacement-independent (i.e. linear). 


\section{A. General expression}

Let us consider a resonator subject to static memoryless nonlinear restoring forces placed in a sine-wave oscillation loop. A noise source $b$ is placed at the input of the resonator. The system is depicted in Fig. 1.

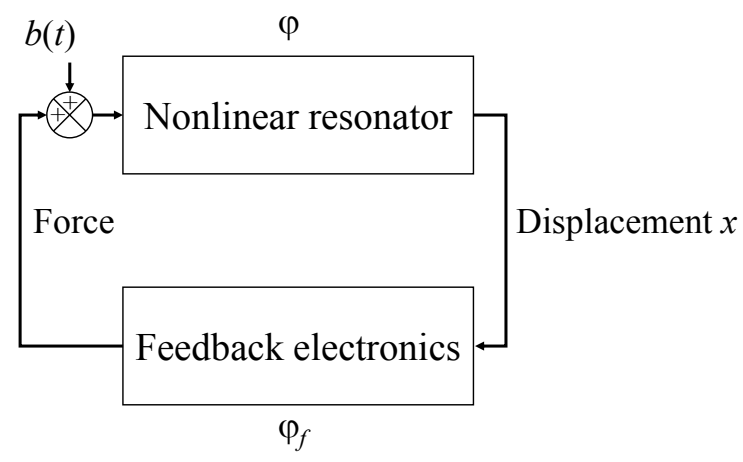

Fig. 1 - Closed-loop circuit of the oscillator. The feedback loop introduces a phase shift $\varphi_{f}$ corresponding to a specific operating point. The condition for selfoscillation is written $\varphi_{f=-} \varphi$.

The behavior of this oscillator may be described by:

$\frac{d^{2} x}{d t^{2}}+\frac{1}{Q} \frac{d x}{d t}+x(1+g(x))=F \sin \left(\omega_{s} t\right)+b(t)$

where $x$ is the normalized displacement of the resonator, $t=\omega_{0} \tau$ where $\omega_{0}$ is the natural frequency of the resonator and $\tau$ is the time variable, $Q$ the $\mathrm{Q}$-factor of the resonator, $\omega_{S}$ the actuation frequency normalized with respect to $\omega_{0}$ and $b$ a stochastic noise. The function $g$ corresponds to a nonlinear restoring force. In (1), $b$ will be considered as a small perturbation to the steady-state nonperturbed regime:

$x_{S}(t)=A_{S} \sin \left(\omega_{s} t+\varphi\right)$

where $\varphi$ is the phase between the actuation force and the displacement.

Since we focus on high-Q resonators, the displacement of the resonator will be restricted to its first harmonic. Let us write:

$x(t)=A(t) \sin (\psi(t))$

The perturbation of the steady-state regime is supposedly small so that we will assume:

$\left|\frac{d^{2} A}{d t^{2}}\right| \ll A \omega^{2}$ and $\left|\frac{d A}{d t}\right| \ll Q A \quad\left(\right.$ with $\left.\omega=\frac{d \psi}{d t}\right)$

The method of harmonic balance applied to (1) yields to:

$\left\{\begin{array}{l}A\left(1+g_{1}(A)-\omega^{2}\right)=F \cos \varphi+b^{s} \\ 2 \frac{d A}{d t} \omega+A \frac{d \omega}{d t}+\frac{A \omega}{Q}=-F \sin \varphi+b^{c}\end{array}\right.$

where

$\left\{\begin{array}{l}g_{1}(A)=\frac{1}{\pi} \int_{t}^{2 \pi+t} g\left(A \sin \psi\left(t^{\prime}\right)\right) \sin \psi\left(t^{\prime}\right) d \psi \\ b^{s}=\frac{1}{\pi} \int_{t}^{2 \pi+t} b\left(t^{\prime}\right) \sin \psi\left(t^{\prime}\right) d \psi \\ b^{c}=\frac{1}{\pi} \int_{t}^{2 \pi+t} b\left(t^{\prime}\right) \cos \psi\left(t^{\prime}\right) d \psi\end{array}\right.$

Since $b$ is a small perturbation, we write: 
$A=A_{s}+\delta A$ and $\omega=\omega_{s}+\delta \omega\left(\delta A<<A_{S}\right.$ and $\left.\delta \omega<\omega_{s}\right)$. Neglecting the second order terms in $\delta A$ and $\delta \omega$ leads to:

$\frac{d \delta \omega}{d t}\left(1+\frac{h}{2}\right)+\frac{\delta \omega}{2 Q}(1+h)=\frac{\frac{d b^{s}}{d t}+\frac{b^{S}}{2 Q}-\omega_{s} h b^{c}}{2 Q F \sin \varphi}$

where

$h=-\frac{F \cos \varphi+\left.A_{s}^{2} \frac{d g_{1}}{d A}\right|_{A_{s}}}{2 Q \omega_{s} F \sin \varphi}$

Assuming that $b_{c}$ and $b_{s}$ are not correlated, the frequency noise spectrum is given by:

$S_{\delta \omega}(\Delta \omega)=\left|T F\left(\frac{\delta \omega}{b^{s}}\right)\right|^{2} S_{b^{s}}+\left|T F\left(\frac{\delta \omega}{b^{c}}\right)\right|^{2} S_{b^{c}}$

where $S_{b^{s}}$ (respectively $S_{b^{c}}$ ) is the spectrum of $b_{s}$ (respectively $b_{c}$ ).

One can notice from (9) that the spectrum of the oscillator is the sum of two components respectively related to the spectrum of the in-phase and quadrature components of the injected noise. This is coherent with the interpretation of the LTV approach developed by Hajimiri and Lee [5]. To calculate $\mathrm{S}_{\delta \omega}(\Delta \omega)$ in the case of white noise, we may write:

$S_{b^{c}}=S_{b^{s}}=\frac{\sigma_{b}^{2}}{\pi}$

where $\sigma_{b}$ is the standard deviation of $b$.

Finally, the optimal performance of the oscillator can be derived by studying the variance of $\delta \omega$, which is calculated through:

$\sigma_{\delta \omega}^{2}=2 \int_{0}^{+\infty} S_{\delta \omega}(\Delta \omega) d(\Delta \omega)$

The actuation force of the resonator in a MEMS oscillator always has a component in quadrature with the displacement (otherwise it cannot oscillate). Hence, we will suppose that $\varphi$ is far from 0 or $\pi$. Since oscillators are usually made of high-Q resonators we will then assume $|h|<<1$, which gives:

$\sigma_{\tilde{\omega} \omega}^{2}=\frac{\sigma_{b}^{2}}{4 \pi Q^{2} F^{2} \sin ^{2} \varphi}\left[1+2 Q \pi h^{2} \omega_{s}^{2}\right]$

To operate the resonator in closed-loop, the phase $\varphi_{f}$ introduced in the feedback loop has to verify:

$\varphi_{f}=-\varphi$.

One can estimate from (12) the optimal closed-loop phase shift $\varphi_{f}$ to minimize the frequency noise. This is illustrated in the Subsection II.B.

\section{B. Case-study of a resonator subject to cubic hardening}

Among the varieties of nonlinearities affecting MEMS resonators [11], cubic hardening models accurately the increasing stiffness of mechanical resonators at large displacement amplitudes [15] [16].

In Fig. 2, we study the behavior of a MEMS resonator subject to a cubic nonlinear restoring force estimated from (14). This situation corresponds to:

$g(x)=\gamma x^{2}$

The illustration is given for $\gamma=0.8, Q=100$ and $\sigma_{b}=10^{-4}$. In order to validate our model, we have performed transient simulations of (1) for different actuation amplitudes $F$ and changed the operating point by choosing the closed-loop phase shift. One can notice, in Fig. 2, that the variance of $\delta \omega$ obtained in transient simulations corresponds to our estimation given by (12). 

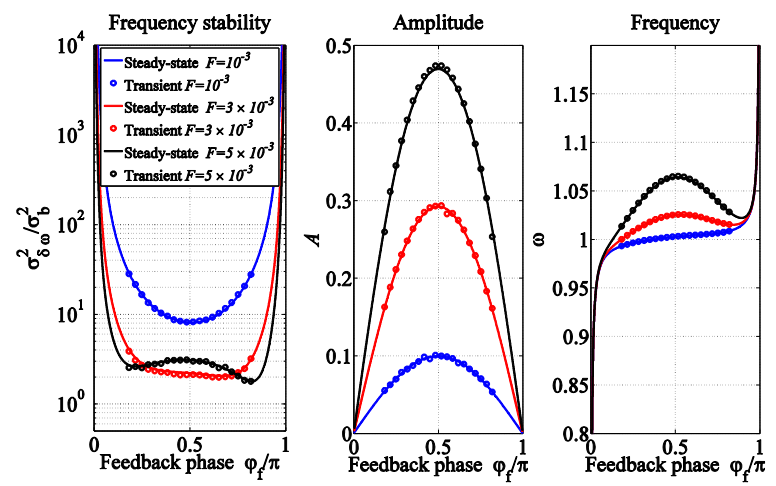

Fig. 2 - Variance of the frequency instability (left), oscillation amplitude (center) and oscillation frequency (right) with respect to the closed-loop phase shift for increasing actuation amplitudes $\left(F=10^{-3}, F=3 \times 10^{-3}\right.$ and $\left.F=5 \times 10^{-3}\right)$. The solid lines correspond to the solutions given by (13) and the circles to transient simulations of (1).

As long as the actuation force and the displacement of the resonator are small, the cubic nonlinearity may be neglected. In this case, the optimal phase shift with respect to, on the one hand, the maximal amplitude and, on the other hand, the frequency noise is located at quadrature (blue lines in Fig. 1).

However, at larger amplitudes, the optimal operating point with respect to the frequency stability starts to shift towards phases far from quadrature. The level at which this phenomenon arises strongly depends on the value of $\gamma$ and $Q$ but it should generally not be misjudged. The same conclusion has been reached by Villanueva et al. [17] where it has been interpreted as the combination of the frequency sensitivity to thermal noise and to the resonator parameter variations (like the quality factor or the Duffing coefficient). However, our analysis shows that the presence of white noise at the input of the MEMS resonator is sufficient to generate a similar effect.

In Section III, we will generalize our method to a nonlinear actuation force and compare the results to the previously studied cubic nonlinear restoring force.

\section{PERFORMANCE OF CAPACITIVE MEMS-BASED OSCILLATORS}

In this section, we estimate the effect of the closed-loop phase shift $\varphi_{f}$ on the frequency stability of a capacitive MEMS-based oscillator. We focus on a one-sided parallel-plate capacitive MEMS resonator. The behavior of such a resonator is known to be affected by a nonlinear actuation force which can be written [15]:

$$
F_{e}(x)=\frac{\eta\left(1+V_{0} \sin \omega t\right)^{2}}{(1-x(t))^{2}}
$$

where $x$ is the displacement normalized with respect to the gap distance between the electrodes, $\eta$ the electromechanical transduction coefficient and $V_{0}$ the amplitude of the actuation voltage normalized with respect to the bias voltage $V_{b}$ applied on the resonator. Since the actuation force is usually small compared to the bias voltage, we will assume $V_{0}<<1$. The existing studies relating to the influence of the closed-loop phase shift on the frequency stability of MEMS oscillators have addressed a cubic nonlinear restoring force and a linear force [11] [17]. In the case of a capacitive MEMS, this would correspond to a third-order Taylor series expansion of $F_{e}$ for $x<<1$, neglecting all the terms in $V_{0} x$ :

$$
F_{e}(x) \equiv F_{T S}(x) \approx \eta\left(1+2 x+3 x^{2}+4 x^{3}\right)+2 \eta V_{0} \sin \omega t
$$

In this case, the approximate behavior of the resonator would be given by:

$\frac{d^{2} x}{d t^{2}}+\frac{1}{Q} \frac{d x}{d t}+x=F_{T S}(x)+b(t)$

One can notice that the second-order term of the Taylor-series expansion has no impact on the first harmonic of the displacement $x$. Thus, the behavior of the electrostatic force at small amplitudes is similar to a cubic nonlinear restoring force described in Section II assuming:

$\gamma=-4 \eta$ and $F=2 \eta V_{0}$

However, at larger amplitude (i.e. when $x<<1$ does not hold), we have to model the system described by: 
$\frac{d^{2} x}{d t^{2}}+\frac{1}{Q} \frac{d x}{d t}+x=\frac{\eta\left(1+2 V_{0} \sin \omega t\right)}{(1-x(t))^{2}}+b(t)$

In this more complex case, the method of harmonic balance detailed in Section II leads to the results presented in Fig. 3

where we compare the results obtained with the Taylor-Series approximation (17) to the ones obtained from the complete expression of the electrostatic force (19). The results are plotted for $\gamma=-4 \eta=-0.04$ and $Q=100000$ which highlight the differences.
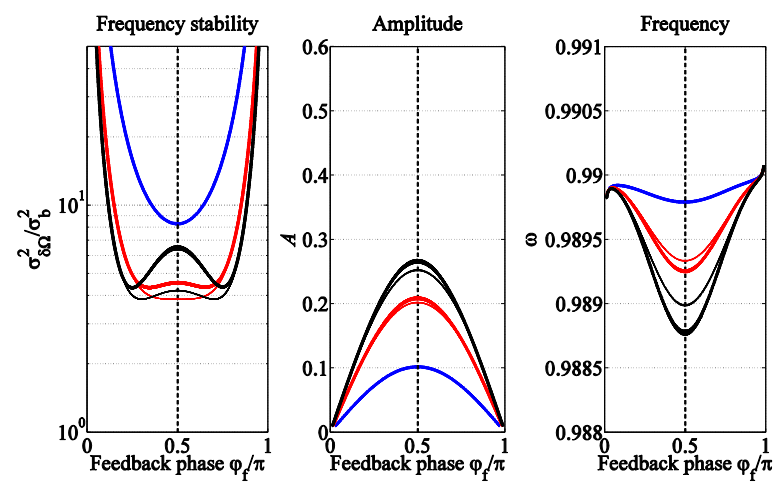

Fig. 3- Variance of the frequency instability (left), oscillation amplitude (center) and oscillation frequency (right) with respect to the feedback phase for increasing actuation force amplitudes $\left(F=1 \times 10^{-6}\right.$ (blue), $F=2 \times 10^{-6}$ (red) and $F=2.5 \times 10^{-6}$ (dark)). The thick lines correspond to the model described by (20) whereas the thin lines relate to the third-order series expansion (17).

As shown in Fig. 3, a similar phenomenon as in Fig. 2 appears, where the best operating point with respect to the frequency stability is not always located at quadrature. However, Fig. 3 highlights that a cubic nonlinear behavior largely underestimates the specific behavior of a capacitive MEMS. Surprisingly, this phenomenon may arise at relatively low amplitudes (e.g. $20 \%$ of the gap distance here).

The position of the optimal operating point depends on the resonator characteristics such as the electromechanical transduction coefficient $\eta$ and the quality factor $Q$. If the phase shift introduced in the closed loop is not designed properly, improving the signalto-noise ratio (SNR) by increasing the actuation voltage would lead to poor phase noise performance (see local maximum on the solid dark line in Fig. 3). However, a good estimation of the characteristics of the system makes the operation in large amplitude and low phase-noise regimes possible by precisely tuning the closed-loop phase shift.

\section{CONCLUSION}

In this paper, we obtained a closed-form expression to estimate the performance of MEMS oscillators in terms of frequency stability. We found out that, with adequate precautions, driving a capacitive high-Q resonator in closed loop into large amplitude regimes may improve the phase noise performances of the oscillator.

The only noise source which has been considered in this work is a white noise (typically thermal noise) placed at the input of the MEMS oscillator. Obviously, the existence of other noise sources, such as flicker or white electronic noises coming from other components placed inside the loop would deteriorate the performance of the sensor. Hence, our study models a best-case scenario which could be interpreted as a fundamental limit. Studying the impact of the noise coming from detection and/or amplifier stages on the global performance of the whole system is the subject of ongoing work. 


\section{REFERENCES}

[1] X. Zou and A. Seshia, "A high-resolution resonant MEMS accelerometer," 18th International Conference on Solid-State Sensors, Actuators and Microsystems (TRANSDUCERS), pp. 1247 - 1250, 2015.

[2] U. Krishnamoorthya, R. Olsson, G. Bogartb, M. Bakera, D. Carrb, T. Swilera and P. Clewsa, "In-plane MEMS-based nano-g accelerometer with subwavelength optical resonant sensor," Sensors and Actuators A, Vols. 145-146, pp. 283-290, 2008.

[3] P. Ward and A. Duwel, "Oscillator Phase Noise: Systematic Construction of an Analytical Model Encompassing Nonlinearity," IEEE Transactions on Ultrasonics, Ferroelectrics, and Frequency Control, vol. 58, no. 1, pp. 195-205, 2011.

[4] D. Leeson, "A Simple Model of Feedback Oscillator Noise Spectrum," RFIC Virtual Journal, IEEE, vol. 54, no. 2, pp. 329-330, 1966.

[5] A. Hajimiri and T. H. Lee, "A General Theory of Phase Noise in Electrical Oscillators," IEEE Journal of Solid-State Circuits, vol. 33, no. 2, pp. 179-194, 1998.

[6] X. Sun, R. Horowitz and K. Komvopoulos, "Stability and Resolution Analysis of a Phase-Locked Loop Natural Frequency Tracking System for MEMS Fatigue Testing," Journal of Dynamic Systems, Measurement and Control, vol. 124, pp. 599-605, 2002.

[7] K. L. Ekinci, Y. T. Yang and M. L. Roukes, "Ultimate limits to inertial mass sensing based upon nanoelectromechanical systems," Journal of Applied Physics, vol. 95, no. 5, pp. 2682-2689, 2004.

[8] V. Kaajakari, J. K. Koskinen and T. Mattila, "Phase Noise in Capacitively Coupled Micromechanical Oscillators," IEEE Transactions on Ultrasonics, Ferroelectrics, and Frequency Control, vol. 52, no. 12, pp. 2322-2331, 2005.

[9] D. K. Agrawal and A. A. Seshia, "An Analytical Formulation for Phase Noise in MEMS Oscillators," IEEE Transactions on Ultrasonics, Ferroelectrics and Frequency Control, vol. 61, no. 12, pp. 1938-1952, 2014.

[10] M. Pardo, L. Sorenson, W. Pan and F. Ayazi, "Phase Noise Shaping via Forced Nonlinearity in Piezoelectrically Actuated Silicon Micromechanical Oscilaltors," in MEMS, Cancun, 2011.

[11] E. Kenig, M. C. Cross, L. G. Villanueva, R. B. Karabalin, M. H. Matheny, R. Lifshitz and M. L. Roukes, "Optimal operating points of oscillators using nonlinear resonators," Physical Review E, vol. 86, no. 5, p. 056207, 2012.

[12] J. Juillard, "A comparative study of reduced-order modeling techniques for nonlinear MEMS beams," in Symposium on Design, Test, Integration \& Packaging of MEMS and MOEMS, Montpellier, France, 2015.

[13] J. Juillard, A. Bonnoit, E. Avignon, S. Hentz and E. Colinet, "Large amplitude dynamics of micro-/nanomechanical resonators actuated with electrostatic pulses," Journal of Applied Physics, vol. 107, p. 014907, 2010.

[14] S. Sancho, F. Ramírez and A. Suárez, "Analysis and reduction of the oscillator phase noise from the variance of the phase deviations, determined with harmonic balance," in Microwave Symposium Digest, 2008 IEEE MTT-S International, Atlanta, 2008.

[15] M.-H. Bao, Micro Mechanical Transducers, Elsevier, 2000.

[16] R. Lifshitz and M. C. Cross, "Nonlinear Dynamics of Nanomechanical and Micromechanical Resonators," in Review of Nonlinear Dynamics and Complexity, Wiley, 2008.

[17] L. G. Villanueva, E. Kenig, R. B. Karabalin, M. H. Matheny, R. Lifshitz, M. C. Cross and M. L. Roukes, "Surpassing Fundamental Limits of Oscillators Using Nonlinear Resonators," Physical Review Letters, vol. 110, no. 17, p. 177208, 2013. 